\title{
Abortion During the COVID-19 Pandemic: Racial Disparities and Barriers to Care in the USA
}

\author{
Taida Wolfe $^{1} \cdot$ Yana van der Meulen Rodgers ${ }^{1}$ (i)
}

Accepted: 15 March 2021 / Published online: 22 March 2021

(C) The Author(s), under exclusive licence to Springer Science+Business Media, LLC, part of Springer Nature 2021

\begin{abstract}
This article draws on first-hand experience as well as scholarly research to illuminate how COVID-19 has impacted an already-embattled medical service in the USA, subsequently affecting the reproductive health and experiences of diverse individuals navigating an unfamiliar health and economic environment. COVID-19's introduction into a landscape of abortion restrictions has intensified the barriers that providers and communities already face, with disproportionate impacts on Black and Hispanic abortion seekers. Relaxing existing restrictions on medication abortions and telemedicine delivery models may be one way to ease the tension between keeping people home and getting them the treatment they need.
\end{abstract}

Went to clinic two days ago after a one month absence. In June when many states in this part of the country had not eased their shutdown restrictions, there were at least 10 older white protesters when usually there are 3-4. One large sign that was propped up on the busier street next to the clinic angrily asked why abortion mills were still open when everything else was closed. Today, as I am walking into the clinic with a co-worker, an older white man who is a frequent protestor cautioned us against going inside to kill our Black babies, while a sign proclaiming all lives, black lives, and babies lives matter floated in the background. This clinic is lucky. The escorts - people who guide patients past protest lines - are still here. At other clinics, the lack of escorts has meant protesters are able to walk right up to patients to spew their rhetoric. As I walked into the entryway there were patients and support people sitting in the hallway, which has never happened in the almost ten years I have been working here. The need for social distancing has made clinics think of new ways to service patients who need abortion. I have seen the implementation of pager systems so that patients can wait in their cars, the exclusion of

Yana van der Meulen Rodgers

yana.rodgers@rutgers.edu

Taida Wolfe

tjw104@scarletmail.rutgers.edu

1 Department of Women's, Gender, and Sexuality Studies, Rutgers University, 162 Ryders Lane, New Brunswick, NJ 08901, USA support people in waiting rooms, and fewer patients being scheduled as we adjust to this different, and hopefully temporary, time. (Excerpt from Author's Personal Journals, August 2020).

\section{Introduction}

The COVID-19 pandemic has generated unprecedented health and economics losses as well as disruptions to family lives, employment, school, and interpersonal relationships. It has also exposed the strengths and vulnerabilities of the safety nets upon which different communities rely (Bahn et al., 2020). The healthcare sector, especially abortion care, has experienced particularly severe costs and disruptions. Access to abortion, a healthcare service already long under siege from opponents, has become even more tenuous during a pandemic that has involved widespread shutdowns and shelter-in-place orders. Service providers have also experienced adverse effects from the pandemic, including shortages of personal protective equipment to ensure the safety of essential workers.

The opening journal entry narrates the personal experience of an abortion provider engaged in this work during the pandemic. In this article, we draw on this first-hand experience as well as scholarly research to illuminate how COVID19 has impacted an already-embattled medical service in the USA, subsequently affecting the reproductive health and experiences of diverse individuals navigating an unfamiliar health and economic environment. In situations of economic precarity, many people may decide to delay childbearing, 
thus highlighting the essential nature of contraceptive and abortion access. However, despite the potential increased need, the geography of abortion provision has become even more tenuous during the pandemic as clinics fight to keep their doors open and people struggle to obtain care.

In highlighting the racial disparities and barriers to abortion care in the USA during the pandemic, our article first steps back to provide a brief history of abortion regulations in the USA and disparities in access to care for marginalized populations. Next, we document the additional barriers to abortion care that have resulted from the COVID-19 pandemic and how these obstacles have had differential effects on the decisions of abortion seekers across demographic groups. The final section discusses implications for policy changes and future research.

\section{A History of Disparities in Access to Care}

I came in today, a weekday, to the abortion clinic to begin my day of seeing patients and performing procedures. After I got changed, I headed to the surgical area after picking up the protective gown I wear over my scrubs. I walked down a long hallway that leads to the surgical area. I eventually logged into the computer, after several attempts to remember my password, to look over the list of patients who are ready. I notice that it is nearing 9:30am and I have a half hour before I must do the mandatory state-directed counseling as required by the state I work in for patients seeking an abortion. The state requires patients to receive this information at least 24 hours before they can have an abortion.

I am always frustrated by this phone call even though I have been doing it for a couple of years. Luckily, we are able to do this counseling over the phone in a conference call setting so that women do not have to travel twice to the clinic. Patients used to be able to communicate during the call and ask questions at the end of it. However, lately I am just talking to the air. While the patients can hear me, I cannot hear them. I found out from clinic staff that abortion protestors were calling in pretending to be patients and verbally abusing the doctors making the call as a way to disrupt the session. So now I talk into what seems like a void, my voice oddly echoing back to me. As I began today's session, the words of the script were familiar, "I am going to give you a two-part code, this code is very important so please write it down"; "Your male partner is responsible financially to help you if you choose to continue the pregnancy, even if he offered to pay for the abortion"; and "The state has created materials describing fetal development and social services agencies that offer alternatives to abortion" ments that have nothing to do with knowing about the safety or the risks of the procedure but are more about dissuading women from their decision. Because state lawmakers condescendingly believe that if patients only knew this one thing - that child support exists or how pregnancies develop - they would decide not have an abortion. (Excerpt from Author's Personal Journals, March 2017).

Disparities by race, ethnicity, and sexual identity in health exist in the context of larger systems of social, medical, and economic inequalities that affect marginalized populations. Systemic inequalities in health, differences in insurance coverage, racism, bias within the healthcare system, and continued social and economic inequalities make those people experiencing intersecting oppressions more vulnerable to a number of health disparities. For example, racial and ethnic minorities are at higher risk of death from heart disease, cancer, and diabetes (Nelson, 2002). LGBTQ people are more likely than straight people to be uninsured, face discriminatory treatment in the healthcare system, and lack access to inclusive sex education (Dawson \& Leong, 2020). Disparities in unintended pregnancy and contraceptive use reflect disparities in abortion rates for racial and ethnically marginalized populations, with Black and Hispanic women being overrepresented among abortion patients (Dehlendorf et al., 2013). In 2011, 38\% of pregnancies were unintended for non-Hispanic White women compared to $64 \%$ for non-Hispanic Black women and 50\% for Hispanic women (Finer \& Zolna, 2016). Queer people who can get pregnant are also more likely to experience unintended pregnancy than straight people and more likely to have an abortion (Dawson \& Leong, 2020). Restrictions on abortion exacerbate these existing disparities in unintended pregnancy rates for marginalized communities.

Although the 1973 Supreme Court decision Roe v. Wade legalized abortion, in the past few decades, most states have implemented regulations curtailing abortion access in some way-promoting abortion scarcity while producing the abortion clinic as a dangerous place in need of state control. Such anti-abortion restrictions include requiring parental consent for minors who seek the service, requiring abortion facilities and doctors to meet the same strict requirements as hospital surgical centers, mandating that abortion seekers receive counseling about the procedure and fetal development, and requiring abortion seekers to wait between 24 and $72 \mathrm{~h}$ after counseling to have an abortion (Daniels et al., 2016). Since Roe v. Wade, anti-abortion forces have used narratives of danger, immorality, and protection to implement a set of restrictions on abortion that justify state surveillance of abortion clinics far beyond what other outpatient clinics have to endure. 
States where people have extremely limited access to abortion are generally Republican dominated and have a strong Christian right influence, and they have fewer women legislators than states with more liberal abortion access (di Mauro \& Joffe, 2007). Restrictive abortion laws have contributed to service reductions and clinic closures nationwide. In 2017, 89\% of US counties had no abortion provider within their borders-up from 77\% in 1978 (Henshaw, 1998; Jones et al., 2019). As of 2018, in 27 US cities, individuals had to travel over 100 miles to reach an abortion clinic (Cartwright et al., 2018). Legal actions during the pandemic intensified such geographical discrepancies in access to abortion. For example, in Texas, the average distance to travel to obtain an abortion rose from 12 to 243 miles when services were shut down during the pandemic (Cappello, 2020).

Abortion providers have also faced a complex web of challenges due to the legal restrictions as well as anti-abortion protestors. For example, TRAP (Targeted Regulation of Abortion Providers) laws requiring abortions to be performed in ambulatory surgical centers could cost an individual facility close to US\$1 million to conduct physical renovations; as such, these costly requirements may result in providers stopping abortions or significantly raising the prices to make quality abortion care beyond some people's reach (Jones \& Weitz, 2009). Restrictions on Medicaid and low Medicaid reimbursement rates have also had negative impacts on providers. To minimize the bite of Medicaid's insufficient reimbursement rates, providers have depended on abortion funds, minimized their work with Medicaid, or avoided Medicaid completely by absorbing large sums themselves in free or reduced-cost services (Kacanek et al., 2010). Abortion providers have also needed to deal with harassment, vandalism, and outright violence (ranging from bombings to arson to chemical attacks) from anti-abortion protesters. Evidence indicates that an increase in the likelihood of harassment or of violence by $10 \%$ reduces the number of abortion providers per 100,000 pregnancies by $7.8 \%$ and $5.9 \%$, respectively, and that these acts are more common in western states and in states with higher levels of sexual violence against women (Medoff, 2015).

The shrinking geography of abortion provision carved out by the legislative and religious campaigns of abortion opponents increasingly pushed abortion out of reach for people of color and low income people. Marginalized populations often bore the financial burden of an unjust reproductive health landscape created by biased policies, especially anti-abortion regulations such as restrictions on Medicaid coverage (Harris \& Wolfe, 2014). These policies often resulted in difficult choices between paying bills and having an abortion, longer travel times and distances to obtain an abortion, and delays in obtaining an abortion because of having to work or organize childcare (Karasek et al., 2016; Howell, 2020; Fuentes et al., 2016). Ethnographic research shows that before the pandemic, Black women often became frustrated with the long wait associated with obtaining an abortion because of having to manage childcare (Wolfe, 2021). COVID-19's introduction into this landscape of abortion constraints has intensified the barriers that many people already faced, as Black and Hispanic individuals are already disproportionately impacted by abortion restrictions (Jerman et al., 2016; Coles et al., 2010).

\section{Barriers and Restrictions During the Pandemic}

Work today was interesting. We have continued to adjust to the COVID-19 pandemic with social distancing implemented in the waiting room so that chairs are not as tightly packed. As I walked the protest line, I did not wear my earphones today and one protester who is there regularly whispered to me as I was walking by, "It's terrible that you are killing your own people," of course referencing my race and the fact that Black people are overrepresented among abortion patients. This was not so much of a surprise as recent campaigns by abortion opponents have focused on the use of abortion within Black communities, trading on the history of state-sanctioned reproductive oppression of poor communities and communities of color that has been part of United States history. But abortion opponents are not interested in reproductive justice nor the wishes of Black folk, they are seeking to weaponize shame. Once inside, I put on my scrubs and opened the box of the face shields that I just ordered off the internet to put on in addition to my mask. It often gets hot underneath all the protective layers, and condensation builds--fog and little droplets routinely appear on the face shield. As I move through the day I often have to remind patients to either put their mask back on or pull it up over their noses. Some even ask if they have to keep their masks on during the procedure. Others when confronted about mask wearing argue they cannot breath even as I sit there under layers of protective gear, some of which I have had to purchase myself. (Excerpt from Author's Personal Journals, January 2021).

COVID-19 has exacerbated the barriers that people face in trying to either practice or access abortion care. Social distancing requirements and the lack of childcare in the midst of school shutdowns have placed limits on staffing capacity and the number of patients that clinics can schedule. These barriers exist even in "abortion friendly" cities. Abortion providers and seekers have also faced legal restrictions, especially in the South. Already experiencing hostile environments before the pandemic, clinics faced the 
possibility of closing their doors as 12 state governments attempted to stop abortion services in March and April 2020, declaring the service to be "nonessential" during coronavirus business closures (Ruggiero et al., 2020). The inclusion of abortion on the list of nonessential services was inappropriate and legally contested, with court orders blocking these bans in some of the states (including Alabama, Ohio, Oklahoma, and Tennessee) and allowing abortions to continue (ACOG, 2020b; Baker, 2020). In some states such as Texas and Arkansas, parts of the executive orders did take effect and abortions that were not immediately medically necessary were effectively prohibited. By the end of May 2020 , these bans had either been lifted or allowed to expire (Sobel et al., 2020).

In addition to the legislative barriers, financial constraints during COVID-19 worked to shrink abortion provision as the federal government treated abortion clinics differently from anti-abortion crisis pregnancy centers. The government obligated Planned Parenthood to return approximately US\$60 million in PPP (Paycheck Protection Program) loans while funneling anywhere from US $\$ 4$ to 10 million to anti-abortion crisis pregnancy centers that routinely disseminate false information to people seeking abortion care (Rosen, 2012). This biased fiscal policy response endangers abortion access as providers struggle to keep their doors open during an economic crisis while also fighting antiabortion campaigns that are increasingly supported by the federal government (Glenza, 2020).

Officials in the states attempting to block access to abortion during COVID-19 argued that restricting abortions would free up medical supplies and personnel by postponing elective procedures until the crisis is over. However, abortion is a time-sensitive service both in terms of health and in terms of legal restrictions-in the majority of states, abortion cannot be performed after 24 weeks of pregnancy. As previous research has shown, restricting reproductive healthcare has adverse effects for women (Rodgers, 2018; Ralph et al., 2019). Forcing those who are pregnant to delay an abortion endangers both their physical health and their economic future.

Individuals who have abortions in the second trimester face a greater risk of hemorrhage and other complications like uterine perforation, which may require further surgery or even a hysterectomy to address (Darney et al., 1990; ACOG, 2020b; Upadhyay et al., 2015). Delays could also extend the pregnancy to the point of fetal viability, after which most states prohibit abortions except to protect the life and health of the woman. There are also financial consequences when women are forced to carry an unwanted pregnancy to term: women denied an abortion because of restrictive state laws are less likely to be employed full-time for years afterwards than women who got the abortion they needed, and they are more likely to live in poverty after being denied an abortion (Foster et al., 2018). Such adverse economic effects from an unwanted pregnancy would compound any other hardships facing a new mother in an economy decimated by pandemic-related layoffs and business closures.

Abortion providers have also faced difficulties during the pandemic, which arose not only from the state-mandated restrictions on abortion services but also from patientrelated reasons, especially cancelled or postponed appointments, and personnel-related reasons, especially those that prevented clinicians and staff from going to work. Evidence from a survey of 103 independent abortion clinics in April-May 2020 indicates that clinicians and staff members experienced disruptions in their work due to the pandemic at half of the clinics surveyed (Roberts et al., 2020). Among the personnel-related reasons, the main reasons for these work stoppages were quarantine restrictions, belonging to a high-risk group, getting sick with the virus, and childcare responsibilities. Of note, and consistent with the author's personal experience, some respondents reported growing harassment against staff and patients by protesters at their clinics, and they reported the need to hire more security guards. Another tactic used by protesters was to report clinicians to the Department of Health for COVID violations (Roberts et al., 2020).

The COVID-19 pandemic has also forced abortion providers to think of novel ways to service patients that allow for social distancing, protection of workers and patients, and adherence to the regional restrictions on abortion care. Survey evidence indicates that already in the early months of the pandemic (March-May 2020), 71\% of independent abortion clinics had shifted to telehealth for follow-up appointments, $41 \%$ had moved to telehealth for consultations, and up to $43 \%$ had reduced their testing (Upadhyay et al., 2020). In addition to remote services, clinics have implemented asynchronous schedules or have extended the workday to spread out appointments (Roberts et al., 2020). Some clinics have had to suspend reproductive services including contraceptive visits and testing for sexually transmitted infections. Over $70 \%$ of clinics operating in states that ruled abortion to be a non-essential service had to cancel, postpone, or even temporarily close abortion services (Roberts et al., 2020).

Abortion remains an essential service in numerous states hard hit by COVID-19. In principle, people in states with COVID-related abortion bans could obtain the service in neighboring states. However, distance as a barrier to abortion disproportionately affects low-income individuals who lack the financial resources and time to travel. Another option is greater reliance on medication abortions (non-surgical abortion services). However, until July 2020, the Food and Drug Administration (FDA) still required that mifepristone, the first of two drugs used in medication abortions, be dispensed in a clinic, hospital, or 
medical office. This requirement for in-person dispensing of mifepristone posed a threat not only to abortion seekers but also to clinicians, and it had disproportionately negative effects on communities of color already hard hit by the pandemic (ACOG, 2020a). The requirement, combined with an increasingly restrictive abortion environment, also contributed to a greater likelihood of individuals self-managing their abortions in unsafe ways (Harris $\&$ Grossman, 2020). The remainder of 2020 saw extensive legal challenges to this requirement, as we discuss in the concluding section.

COVID-19 has exacerbated the ever-present disparities in access to abortion care despite many people changing their plans to have children because of the pandemic. During the pandemic, over $40 \%$ of cisgender women in a US survey indicated that due to COVID-19, they had changed their plans about the timing of pregnancy or how many children to have. This average hides considerable differences by race/ethnicity: $44 \%$ of Black women and $48 \%$ of Hispanic women wanted to delay having children or have fewer children, compared to $28 \%$ of White women (Lindberg et al., 2020). Disparities were also found along sexuality and class dimensions: queer women were more likely than straight women to want to delay or have fewer children (46 versus 33\%), and low-income women were more likely than higher-income women to report changes in childbearing preferences (37 versus 32\%) (Lindberg et al., 2020). These figures highlight how the reproductive needs and choices of marginalized populations have been more susceptible to change during the pandemic. Such choices may raise the demand for abortion services precisely when financial precarity and austerity increasingly dictate the capacity of clinics to provide abortion services.

Not only has the pandemic altered planning around pregnancy, but it also has contributed to a spike in intimate partner violence (IPV). IPV and other forms of domestic violence intensify during disasters and crises as tensions mount within households from the health issues and associated economic insecurity (Gearhart et al., 2018). The COVID-19 crisis is longer term, more people are confined to their homes, there is an uncertain endpoint, many are struggling financially, and people are scared and grieving. It is difficult to overstate the scale of this problem for those who are subject to abuse of all kinds. Extensive data from daily mobile device tracking as well as police reports and crime data to show that shelter-in-place orders in the USA caused domestic violence to increase by approximately $6 \%$ (over 24,000 cases) from mid-March to lateApril, 2020 (Hsu, 2021). Previous research has linked IPV to unplanned pregnancies (Miller et al., 2010), with the implication that the stay-at-home measures and spike in IPV during the pandemic could increase the need for abortion access.

\section{Moving Forward}

A global pandemic superimposed on an increasingly restrictive abortion environment makes it vital to theorize and research novel approaches to abortion care that attend to the structural barriers, political obstacles, and racial disparities in reproductive healthcare that affect individuals' ability to manage their fertility. COVID-19 has also forced medical professionals to think of new ways to manage healthcare. Abortion providers have implemented a number of protocols, including telemedicine, designed to provide care given legislative restrictions and the social realities of the COVID-19 pandemic.

Many of these protocols, built around medication abortion, are designed to keep both patients and workers safe while still providing high-quality care to their communities. The urgency of providing care in the midst of a pandemic has revived interest in and has expanded telemedicine delivery models as a mechanism for increasing access to abortion services. For example, in April 2020, a number of organizations including Gynuity Health Projects and the Society of Family Planning created a no-touch protocol that significantly expands on current telemedicine delivery models. This protocol permits medication abortions without the need for in-clinic pregnancy testing, pre-procedure labs, or ultrasounds (Raymond et al., 2020). However, despite the exciting possibilities that delivery models for telemedicine offer, abortion providers are often burdened by federal and state restrictions that require a physician to dispense mifepristone in person. As a result, many clinics have not been able to adopt such measures (Upadhyay et al., 2020). Currently, 18 states prohibit telemedicine for abortion in some way, with 5 states completely prohibiting it and 13 requiring that a physician be physically present with the patient during the procedure. In addition, 14 states require a person to receive an ultrasound before an abortion while 13 require in-person counseling before a procedure. These policies effectively bar no-touch or other telemedicine protocols from being implemented, thus exacerbating barriers to care (Ramaswamy et al., 2020).

The pandemic has ushered in a major legislative change in the provision of medication abortions that has eliminated the in-person dispensing requirement in a number of states. In July 2020 in response to a legal suit filed by the American College of Obstetricians and Gynecologists and other organizations, a federal court blocked the FDA's enforcement of the in-person dispensing of mifepristone, thus allowing eligible patients in states that allow telemedicine abortion to obtain mifepristone and misoprostol from providers directly through the mail or through a mail-order pharmacy. Some states have seen new standalone telemedicine services through start-up non-profit 
organizations that provide mail-order medication abortions. After the July 2020 ruling, the Trump administration unsuccessfully tried to reinstate the in-person dispensing requirement and ultimately turned to the Supreme Court, where the matter remained on hold through the rest of 2020 (Baker, 2020; SCOTUS, 2020). However, on January 12, 2021, in the decision ACOG v. FDA, the Supreme Court effectively blocked the ability of abortion providers to send mifepristone through the mail, thus preventing many clinics from limiting person-to-person contact while providing necessary care (SCOTUS, 2021).

More research is needed on people's preferences and experiences with self-managed abortion across the globe, the best potential methods of distribution of abortion medications such as mifepristone, protocols for assessing eligibility for medication abortion given gestational limits, and the clinical networks needed to help people assess and manage complications without shame (Moseson et al., 2020; Kapp et al., 2018). Also needed is more scholarship on how the pandemic has affected the reproductive health and abortion experiences for marginalized populations in the USA - communities who already face disparities in access to healthcare due to historic and contemporary systems of social and economic inequality.

Relaxing existing restrictions on medication abortions in states that still have them and increasing advocacy efforts around eliminating legislative roadblocks to telemedicine delivery methods will go a long way to ease the tension between keeping people home and getting them the treatment they need. Fewer restrictions on medication abortions will also support abortion providers doing this work during and after the pandemic by reducing the various risks that they face, including exposure to the virus, financial hardships, and harassment at in-person clinics. Such measures have the potential to greatly reduce traditional racial, economic, and geographic barriers to abortion care.

Went to clinic today. Was interesting seeing how COVID continues to impact service and the old ways we 'do' clinic. Today, there was only one protestor standing outside quietly with his signs on the ground face up, as the parking lot on the other side of the street no longer permits protestors to hang their signs on the fence. As I got dressed for the day, I saw new faces and couldn't find older ones. Staff turnover is a constant thing when not in a pandemic, as administrative and nursing staff and medical assistants move in and out of this work for various reasons. One medical assistant who had been here for some time left during COVID, it was explained to me, because she was having issues with childcare. Except for a couple of long-term people who have been working there longer than me, most of the medical assistants are newish and, as always, mostly of color. Despite this volatility of staffing, the day proceeded pretty normally. The amount of patients I am seeing at a given in-person clinic is slowly creeping back up to pre-COVID levels as clinics become used to how to schedule patients given social distancing requirements and as states slowly open up. Telehealth during this pandemic has been instrumental for seeing patients at another clinic I work at, allowing us to see more patients than we would be able if we only had in-person appointments.

A Black patient today told me she thought I would be a white lady. Although few say it out loud, I see varying reactions to my race, with some delighted at my being a Black woman, and others completely surprised. In the past, one patient stated she thought I was going to be a white Jewish man, her ideas very specific about the racial, gender, and religion of abortion providers. These ideas of who is an abortion provider are part of the public imagination. A medical assistant at another clinic stated that the protestors know that the other doctor, who is white, is the provider, and they target that person as such. Race, gender, and perhaps religion play a part in who we imagine abortion providers to be and who the abortion patient is. These deeply rooted imaginaries still take hold of patients and protestors alike even when the world has seemingly turned upside down during the pandemic. (Excerpt from Author's Personal Journals, August 2020).

\section{Declarations}

Ethical Approval The project did not involve research with human subjects and did not require IRB approval.

Informed Consent The project did not involve research with human subjects and did not require informed consent.

Conflict of Interest The authors declare no competing interests.

\section{References}

ACOG. (2020a). Court's order lifting burdensome FDA restriction: What you need to know. American College of Obstetricians and Gynecologists.

ACOG. (2020b). Joint statement on abortion access during the COVID-19 outbreak. American College of Obstetricians and Gynecologists.

Bahn, K., Cohen, J., \& Rodgers, Y. (2020). A feminist perspective on COVID-19 and the value of care work globally. Gender, Work \& Organization, 27(5), 695-699.

Baker, C. N. (2020). Abortion regulation in the age of COVID-19. The Regulatory Review, 9(21), 1-6. 
Cappello, O. (2020). Surveying state executive orders impacting reproductive health during the COVID-19 pandemic. Guttmacher Institute.

Cartwright, Alice F., Karunaratne, Mihiri, Barr-Walker, Jill, Johns, Nicole E., \& Upadhyay, Ushma D. (2018). Identifying national availability of abortion care and distance from major US cities: Systematic online search. Journal of Medical Internet Research, 20(5), e186.

Coles, M. S., Makino, K. K., Stanwood, N. L., Dozier, A., \& Klein, J. D. (2010). How are restrictive abortion statutes associated with unintended teen birth? Journal of Adolescent Health, 47(2), $160-167$.

Daniels, C. R., Ferguson, J., Howard, G., \& Roberti, A. (2016). Informed or misinformed consent? Abortion policy in the United States. Journal of Health Politics, Policy and Law, 41(2), 181-209.

Darney, P. D., Atkinson, E., \& Hirabayashi, K. (1990). Uterine perforation during second-trimester abortion by cervical dilation and instrumental extraction: A review of 15 cases. Obstetrics Gynecology, 75(3 Pt 1), 441-4.

Dawson, R., \& Leong T. (2020). Not up for debate: LGBTQ people need and deserve tailored sexual and reproductive health care. Guttmacher Institute.

Dehlendorf, C., Harris, L. H., \& Weitz, T. A. (2013). Disparities in abortion rates: A public health approach. American Journal of Public Health, 103(10), 1772-1779.

di Mauro, D., \& Joffe, C. (2007). The religious right and the reshaping of sexual policy: An examination of reproductive rights and sexuality education. Sexuality Research \& Social Policy, 4(1), 67-92.

Finer, L. B., \& Zolna, M. R. (2016). Declines in unintended pregnancy in the United States, 2008-2011. New England Journal of Medicine, 374(9), 843-852.

Foster, D. G., Biggs, M. A., Ralph, L., Gerdts, C., Roberts, S., \& Glymour, M. M. (2018). Socioeconomic outcomes of women who receive and women who are denied wanted abortions in the United States. American Journal of Public Health, 108(3), 407-413.

Fuentes, L., Lebenkoff, S., White, K., Gerdts, C., Hopkins, K., Potter, J. E., \& Grossman, D. (2016). Women's experiences seeking abortion care shortly after the closure of clinics due to a restrictive law in Texas. Contraception, 93(4), 292-297.

Gearhart, S., Perez-Patron, M., Hammond, T. A., Goldberg, D. W., Klein, A., \& Horney, J. A. (2018). The impact of natural disasters on domestic violence: An analysis of reports of simple assault in Florida (1999-2007). Violence and Gender, 5(2), 87-92.

Glenza, J. (2020). Anti-abortion centers receive at least $\$ 4 \mathrm{~m}$ from US coronavirus bailout. https://www.theguardian.com/world/2020/ aug/03/anti-abortion-centers-paycheck-protection-program

Harris, L. H., \& Grossman, D. (2020). Complications of unsafe and self-managed abortion. New England Journal of Medicine, 382(11), 1029-1040.

Harris, L. H., \& Wolfe, T. (2014). Stratified reproduction, family planning care and the double edge of history. Current Opinion in Obstetrics and Gynecology, 26(6), 539-544.

Henshaw, S. K. (1998). Abortion incidence and services in the United States, 1995-1996. Family Planning Perspectives, 30(6), 263-287.

Howell, M. (2020). Our future must ensure black women can thrive and build our families with dignity. The Washington Informer https:// www.washingtoninformer.com/our-future-must-ensure-blackwomen-can-thrive-and-build-our-families-with-dignity/

Hsu, L. C. (2021). The effect of social distancing on police reports of domestic violence. Feminist Economics 27 (1/2).

Jerman, J., Jones, R. K., \& Onda, T. (2016). Characteristics of U.S. abortion patients in 2014 and changes since 2008. New York: Guttmacher Institute.

Jones, B. S., \& Weitz, T. A. (2009). Legal barriers to second-trimester abortion provision and public health consequences. American Journal of Public Health, 99(4), 623-630.
Jones, R. K.., Witwer, E., \& Jerman, J. (2019). Abortion incidence and service availability in the United States, 2017. Guttmacher Institute.

Kacanek, D., Dennis, A., Miller, K., \& Blanchard, K. (2010). Medicaid funding for abortion: Providers' experiences with cases involving rape, incest and life endangerment. Perspectives on Sexual and Reproductive Health, 42(2), 79-86.

Kapp, Na., Blanchard, K., Coast, E., Ganatra, B., Harries, J., Footman, K., Moore, A., Owolabi, O., Rossier, C., \& Shellenberg, K. (2018). Developing a forward-looking agenda and methodologies for research of self-use of medical abortion. Contraception, 97(2), 184-188.

Karasek, D., Roberts, S. C., \& Weitz, T. A. (2016). Abortion patients' experience and perceptions of waiting periods: Survey evidence before Arizona's two-visit 24-hour mandatory waiting period law. Women's Health Issues, 26(1), 60-66.

Lindberg, L. D., VandeVusse, A., Mueller, J., \& Kirstein, M. (2020). Early impacts of the COVID-19 pandemic: Findings from the 2020 Guttmacher Survey of Reproductive Health Experiences. Guttmacher Institute.

Medoff, M. H. (2015). The impact of antiabortion criminal activities and state abortion policies on abortion providers in the United States. Journal of Family and Economic Issues, 36(4), 570-580.

Miller, E., Decker, M. R., McCauley, H. L., Tancredi, D. J., Levenson, R. R., Waldman, J., Schoenwald, P., \& Silverman, J. G. (2010). Pregnancy coercion, intimate partner violence and unintended pregnancy. Contraception, 81(4), 316-322.

Moseson, H., Herold, S., Filippa, S., Barr-Walker, J., Baum, S. E., \& Gerdts, C. (2020). Self-managed abortion: A systematic scoping review. Best Practice \& Research Clinical Obstetrics \& Gynaecology, 63, 87-110.

Nelson, A. (2002). Unequal treatment: confronting racial and ethnic disparities in health care. Journal of the National Medical Association, 94(8), 666.

Ralph, L. J., Schwarz, E. B., Grossman, D., \& Foster, D. G. (2019). Self-reported physical health of women who did and did not terminate pregnancy after seeking abortion services: A cohort study. Annals of Internal Medicine, 171(4), 238-247.

Ramaswamy, A., Weigel, G., Sobel, L., \& Salganicoff, A. (2020). Medication abortion and telemedicine: Innovations and barriers during the COVID-19 emergency. https://www.kff.org/policy-watch/medication-abortion-telemedicine-innovations-andbarriers-during-the-covid-19-emergency/

Raymond, E. G., Grossman, D., Mark, A., Upadhyay, U. D., Dean, G., Creinin, M. D., Coplon, L., Perritt, J., Atrio, J. M., Taylor, D., \& Gold, M. (2020). Commentary: No-test medication abortion: A sample protocol for increasing access during a pandemic and beyond. Contraception, 101(6), 361-366.

Roberts, S. C., Schroeder, R., \& Joffe, C. (2020). COVID-19 and independent abortion providers: Findings from a rapid-response survey. Perspectives on Sexual and Reproductive Health, 52(4), 217-225.

Rodgers, Y. (2018). The global gag rule and women's reproductive health: Rhetoric versus reality. New York: Oxford University Press.

Rosen, J. D. (2012). The public health risks of crisis pregnancy centers. Perspectives on Sexual and Reproductive Health, 44(3), 201-205.

Ruggiero, S., Brandi, K., Mark, A., Paul, M., Reeves, M. F., Schalit, O., Blanchard, K., Key, K., \& Chandrasekaran, S. (2020). Access to later abortion in the United States during COVID-19: Challenges and recommendations from providers, advocates, and researchers. Sexual and Reproductive Health Matters, 28(1), 1774185.

SCOTUS, Supreme Court of the United States. (2020). Food and Drug Administration v. American College of Obstetricians and Gynecologists. edited by Supreme Court of the United States.

SCOTUS, Supreme Court of the United States. (2021). Food and Drug Administration v. American College Of Obstetricians and Gynecologists. edited by Supreme Court of the United States. 
Sobel, L., Ramaswamy, A., Frederiksen, B., \& Salganicoff, A. (2020). State action to limit abortion access during the COVID-19 pandemic. Kaiser Family Foundation.

Upadhyay, U. D., Desai, S., Zlidar, V., Weitz, T. A., Grossman, D., Anderson, P., \& Taylor, D. (2015). Incidence of emergency department visits and complications after abortion. Obstetrics Gynecology, 125(1), 175-83.

Upadhyay, U. D., Schroeder, R., \& Roberts, S. C. (2020). Adoption of no-test and telehealth medication abortion care among independent abortion providers in response to COVID-19. Contraception: X 2:100049.

Wolfe, T. (2021). Dangerous geographies: On blackness, abortion, and reproductive being: Dissertation in progress. Department of Women's and Gender Studies, Rutgers University.

Publisher's Note Springer Nature remains neutral with regard to jurisdictional claims in published maps and institutional affiliations. 